Case Report

\title{
Lumbar artery pseudoaneurysm following blunt trauma: A case report and literature review
}

\author{
Saurabh Vashisht*, Vikas Bachhal, Vishal Kumar, Deepak Kumar \\ Department of Orthopaedics, Post Graduate Institute of Medical Education and Research (PGIMER), Chandigarh, India
}

\section{A R T I C L E I N F O}

\section{Keywords:}

False aneurysm

Multiple trauma

Computed tomography angiography

\begin{abstract}
A B S T R A C T
Lumbar artery pseudoaneurysms following blunt trauma are very rare. We report a case of polytrauma patient having lumbar spine injury and pelvic fracture presenting with hemodynamic instability. The patient did not improve after aggressive resuscitation. There was no evidence of intraabdominal solid organ or visceral injury. We suspected injury to pelvic vessels as a source of bleeding. Computed tomography (CT) angiography was done. A small size $(6 \times 5 \mathrm{~mm})$ lobulated hyperdense structure is seen in the territory of a left 4th lumbar branch of aorta suggestive of a pseudoaneurysm. Whenever there is a patient of blunt abdominal trauma with pelvic or spine injury and source of bleeding cannot be established, lumbar vessels as a source of bleeding should be kept in mind. CT angiography must be done for diagnosis and patient may subsequently need angioembolization of these vessels.
\end{abstract}

\section{Introduction}

Lumbar artery pseudoaneurysms are rare and usually occur after penetrating injuries [1,2]. Blunt trauma leading to lumbar artery injury and pseudoaneurysms are extremely rare. We report a rare case of polytrauma having a lumbar spine injury, pelvic fractures and bilateral open calcaneus fractures presenting with persistent hypotension and on diagnostic evaluation was found to be a lumbar artery pseudoaneurysm.

\section{Case report}

A 32-year male presented to our hospital after $19 \mathrm{~h}$ of injury with an alleged history of fall from stairs (12 ft). There was a history of abdominal pain, backache and weakness of bilateral lower limbs and the patient was hemodynamically unstable. Resuscitation done, there was free fluid on focused assessment with sonography in trauma (FAST) in the hepatorenal region. After multiple transfusions patient did not improve and was administered inotropes. Afterward, radiographs and Contrast-enhanced computed tomography (CECT) abdomen was done. There was hemoperitoneum without any evidence of solid organ and visceral injury. There were multiple fractures involving bodies of L1, L2; posterior elements of L4, L5 (Figs. 1 and 2); bilateral sacral ala and left side lateral compression injury (Fig. 3). At first, we thought the injury to pelvic vessels as a source of bleeding. CT angiography of the abdomen was done later because of hemodynamic instability and decreasing hemoglobin levels despite multiple packed red blood cell transfusions. A small size $(6 \times 5 \mathrm{~mm})$ lobulated hyperdense (HU 240-250) structure is seen in the territory of a left 4th lumbar branch of aorta suggestive of a pseudoaneurysm (Figs. 4 and 5). We planned for arterial embolization but severely deranged coagulogram

\footnotetext{
* Corresponding author at: 15 Ground Floor, Nehru Hospital, Department of Orthopaedics, PGIMER, Chandigarh 160012.

E-mail address: saurabh90019@yahoo.com (S. Vashisht).
} 


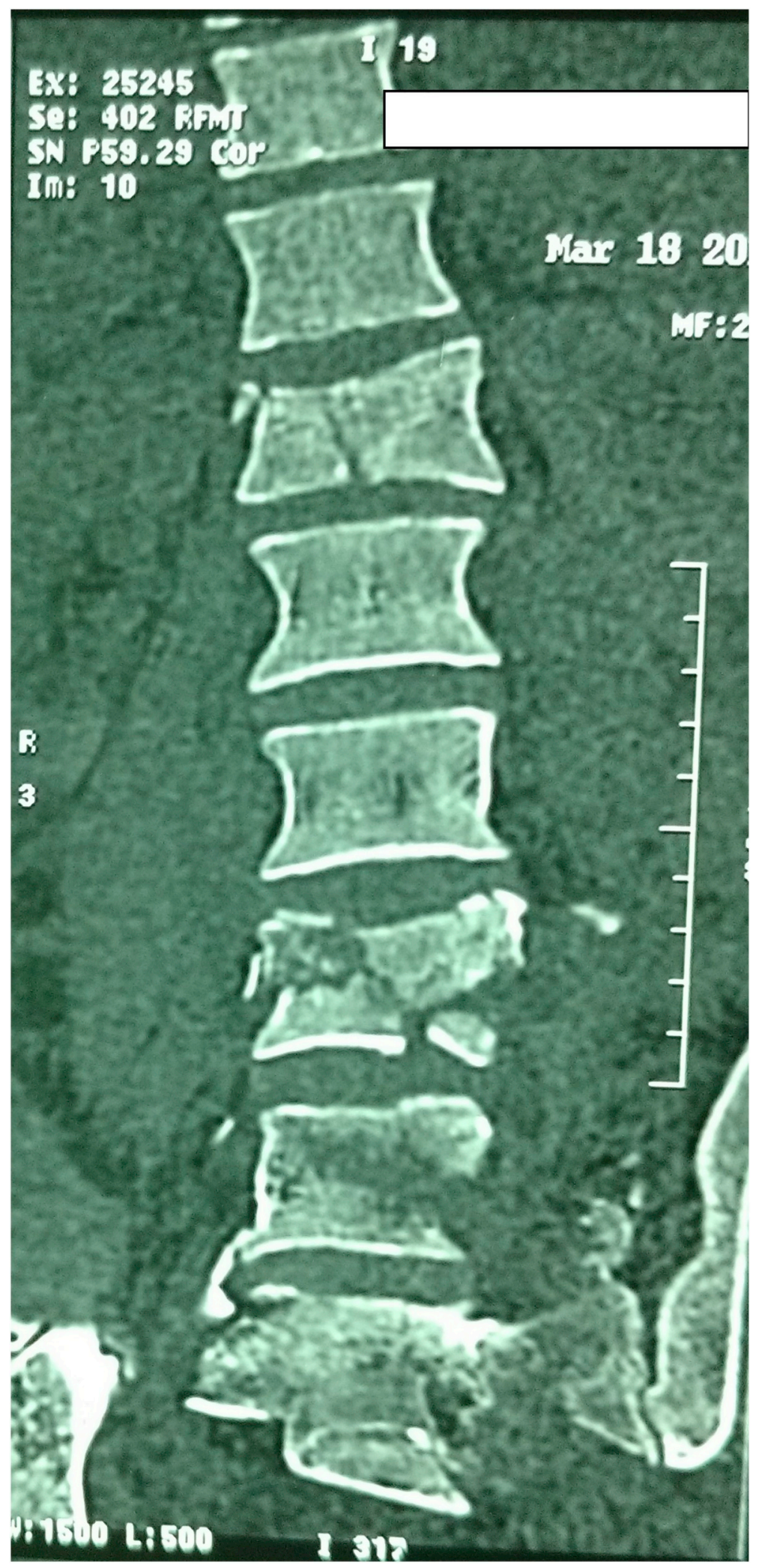

Fig. 1. Coronal CT image showing lumbosacral fractures. 


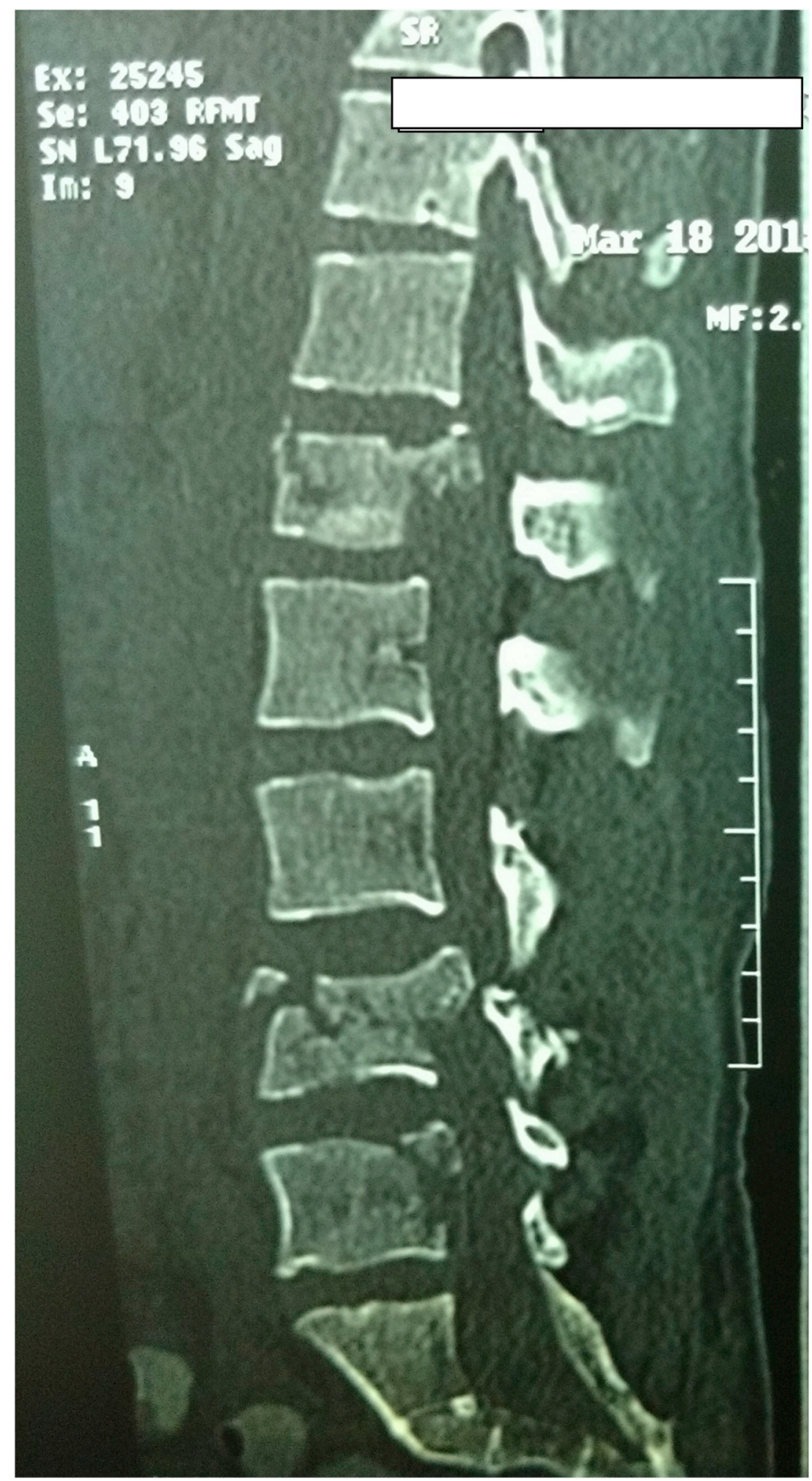

Fig. 2. Sagittal CT image showing lumbar vertebrae fractures. 


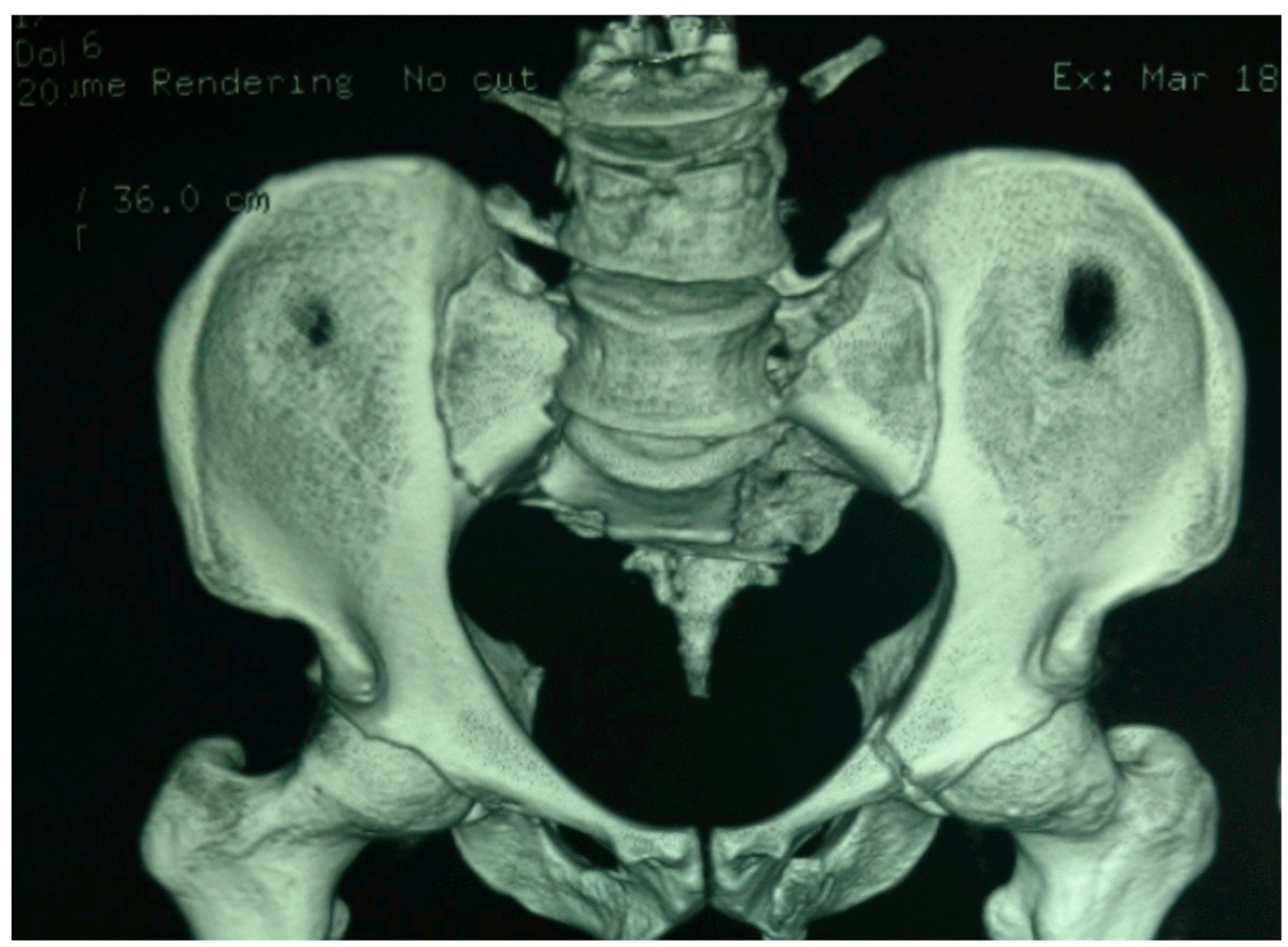

Fig. 3. 3D CT reconstruction images showing pelvic ring injury.

and very low platelet count precluded the intervention. The patient was symptomatically managed with multiple transfusions of packed red cells, platelets, and fresh frozen plasma. Moderate effusion was also present in bilateral pleural spaces with collapse consolidation of underlying lung segments. The patient got shortness of breath on day 5 of admission along with derangement in renal function tests. CT pulmonary angiogram (CTPA) was performed to rule out pulmonary thromboembolism and there was no pulmonary embolism. After that patient responded to symptomatic treatment and improved. Finally, pre-anesthetic clearance was given and debridement of open calcaneus fractures done. Lumbosacral spine injury and pelvic ring injury were managed conservatively. The postoperative period was uneventful and the patient was discharged in hemodynamically stable condition.

\section{Discussion}

There are five pairs of lumbar arteries, first four arise from the posterior aspect of the abdominal aorta and the fifth pair arises from either lateral sacral artery, iliolumbar artery or median sacral artery. These vessels form supply to lumbar vertebrae, adjoining muscles, a distal portion of spinal cord and nerve roots of cauda equine [3,4]. Lumbar artery aneurysms (Table 1) have been reported following penetrating trauma [2], blunt trauma and iatrogenic type including percutaneous kidney procedures, laparoscopic splenectomy [1], lumbar posterolateral fusion [5] vertebral osteomyelitis [6].

These pseudoaneurysms may remain asymptomatic or present with hemodynamic instability. The time of presentation is variable. Early diagnosis is the key as grave complications [7] can occur like increasing size, rupture, hemorrhage, thromboembolism if a diagnosis is missed. Abdominal pain is the primary symptom. Unawareness of this condition and vague symptomatology leads to delay in diagnosis. There should be a high index of suspicion in hemodynamically unstable patients where other causes of retroperitoneal hemorrhage are excluded like bleeding from solid organ injuries, visceral injuries, and major vessel injuries. Psoas abscess, tumors, inflammatory masses can have presentation and symptomatology similar to lumbar artery pseudoaneurysm [3,8].

The diagnosis is also difficult in spinal cord injury patients as a response to injury depends upon the level of lesion and type of injury whether complete and incomplete. The cardinal signs of acute abdominal pathology such as rigidity of abdominal muscles and rebound tenderness tend to be absent in cord lesions above T6 [9]. In our case, there was a history of vague abdominal pain and there was no rigidity and rebound tenderness on per abdominal examination. 


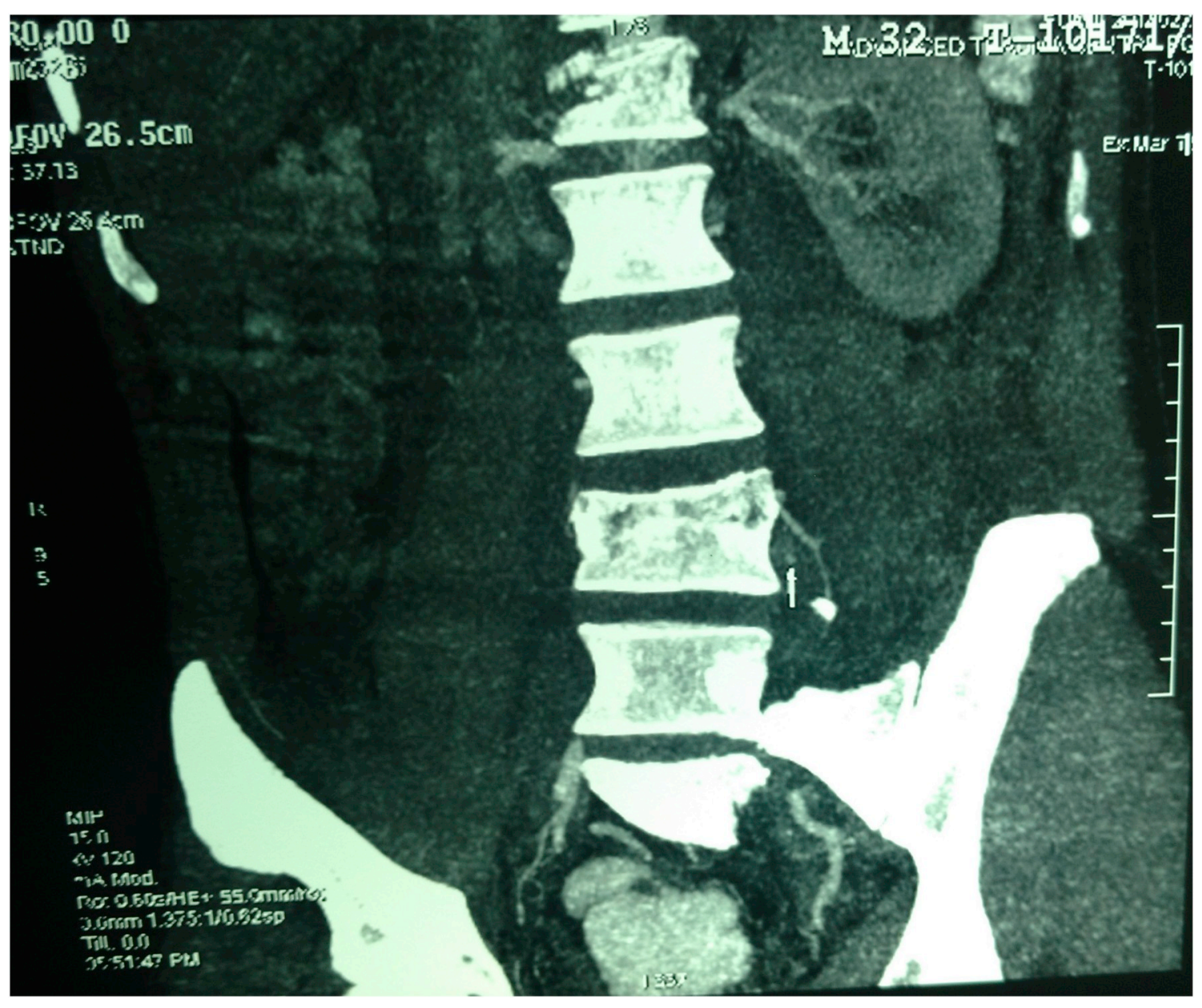

Fig. 4. Coronal CT angiography image showing left 4th lumbar artery pseudoaneurysm.

Radiological investigations are essential in making the diagnosis. The plain radiographs and non contrast CT reveal fractures of lumbosacral spine and pelvis and there should be a high index of suspicion of retroperitoneal hemorrhage in such cases [10]. Lumbar artery pseudoaneurysms appear as increased density mass in close vicinity to the psoas major muscle and the lumen of the aneurysm can be seen on the contrast imaging [1]. Contrast-enhanced tomography is, therefore, more helpful in diagnosing retroperitoneal hematomas, mass lesions and to rule out intraabdominal solid organ injury and visceral injuries [11]. If a patient's condition is deteriorating despite adequate resuscitation and patient is hemodynamically unstable, emergent arteriography is to be done. It gives information about the origin, dimensions, expansion of aneurysm, vascular feeder and decision making for doing any intervention [1].

\section{Conclusion}

So when you encounter a patient of blunt abdominal trauma with pelvic or spine injury and source of bleeding cannot be established, lumbar vessels as a source of bleeding should be kept in mind. In undiagnosed cases, CT angiography must be done for diagnosis and patient may subsequently need angioembolization of these vessels.

\section{Financial disclosures}

None. 


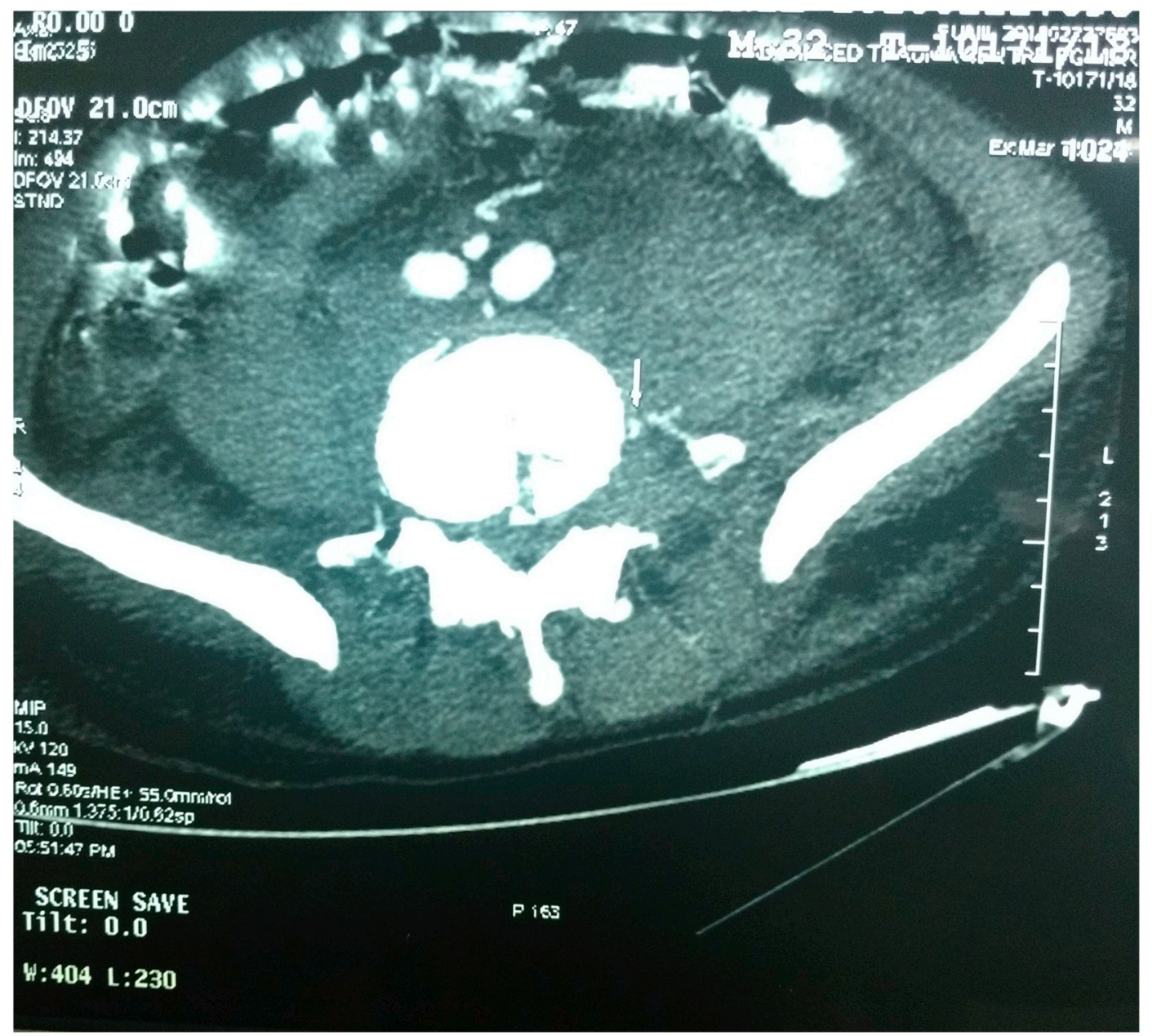

Fig. 5. Axial CT angiography image showing left 4th lumar artery pseudoaneurysm.

\section{Table 1}

Summary of the studies reporting lumbar artery pseudoaneurysms.

\begin{tabular}{|c|c|c|c|c|}
\hline S no. & Author & Year & lumbar artery pseudoaneurysm & Presentation \\
\hline 1 & Ikubo et al & 1993 & Following blunt trauma & $\begin{array}{l}\text { As severe pain abdomen and hypotension (retroperitoneal } \\
\text { hematoma) }\end{array}$ \\
\hline 2 & Chan et al & 2003 & $\begin{array}{l}\text { With traumatic spinal cord injury following gunshot } \\
\text { injury }\end{array}$ & $\begin{array}{l}\text { As severe exacerbation of low back and flank pain during inpatient } \\
\text { rehabilitation. }\end{array}$ \\
\hline 3 & Kessel et al & 2004 & A complication of penetrating abdominal trauma & Delayed presentation and missed during laparotomy \\
\hline 4 & Lee et al & 2008 & With transverse process fracture of the lumbar spine & As hypotensive shock \\
\hline 5 & Tomscot et al & 2013 & $\begin{array}{l}\text { Late complication of osteomyelitis with vertebral body } \\
\text { destruction }\end{array}$ & As acute backache \\
\hline 6 & Oh et al & 2013 & After lumbar posterolateral fusion & As a delayed retroperitoneal hemorrhage \\
\hline 7 & This study & 2018 & With fractures of lumbar spine and pelvis & As hypotensive shock \\
\hline
\end{tabular}

\section{Declaration of Competing Interest}

None.

\section{References}

[1] M. Ceyhan, U. Belet, S. Aslan, S. Ayyildiz, K. Göl, Traumatic lumbar artery pseudoaneurysm: the role of CT angiography in diagnosis and treatment, Diagn Interv Radiol Ank Turk 16 (2) (2010) 162-164 Jun. 
[2] B.J. Kessel, F.A. Habib, B. Thompson, D.E. Bajayo, D. Shatz, Lumbar Artery Pseudoaneurysm, Eur J Trauma 30 (3) (2004) 187-190 Jun 1.

[3] K.-T. Chan, N. Korivi, Lumbar artery pseudoaneurysm in traumatic spinal cord injury: a case report, Arch. Phys. Med. Rehabil. 84 (3) (2003) 455-457 Mar.

[4] J.F. Ratcliffe, The anatomy of the fourth and fifth lumbar arteries in humans: an arteriographic study in one hundred live subjects, J. Anat. 135 (Pt 4) (1982) 753-761 Dec.

[5] Y.M. Oh, H.Y. Choi, J.P. Eun, Delayed retroperitoneal hemorrhage due to lumbar artery pseudoaneurysm after lumbar posterolateral fusion, J Korean Neurosurg Soc 54 (4) (2013) 344-346 Oct.

[6] A. Tomescot, B. Dallaudiere, O. Zurlinden, J. Manelfe, Lumbar artery pseudoaneurysm as a late complication of osteomyelitis with vertebral body destruction, J. Vasc. Surg. 58 (4) (2013) 1084-1087 Oct.

[7] J.S. Lee, C.W. Kim, K.T. Suh, Lumbar artery injury combined with a transverse process fracture of the lumbar spine presenting with hypovolemic shock after a fall - a case report, J Korean Orthop Assoc 43 (3) (2008) 400-403 Jun 1.

[8] D.H. Hulnick, D.P. Naidich, E.J. Balthazar, A.J. Megibow, M.A. Bosniak, Lumbar artery pseudoaneurysm: CT demonstration, J. Comput. Assist. Tomogr. 8 (3) (1984) 570-572 Jun.

[9] Z. Bar-On, A. Ohry, The acute abdomen in spinal cord injury individuals, Paraplegia 33 (12) (1995) 704-706 Dec.

[10] A. Kalangos, B. Walder, B. Faidutti, Ruptured lumbar artery pseudoaneurysm: a diagnostic dilemma in retroperitoneal hemorrhage after abdominal trauma, J. Trauma 45 (4) (1998) 829-832.

[11] A. Ikubo, M. Komura, N. Matoba, T. Fujii, K. Satoh, H. Masuda, et al., Lumbar artery pseudoaneurysm: an unusual cause of a retroperitoneal hematoma: report of a case, Surg. Today 23 (7) (1993) 635-638. 